\title{
Performance of Optical Burst Switching in Time Division Multiplexed Wavelength-Routing Networks*
}

\author{
Tai-Won Um, YoungHwan Kwon, and Jun Kyun Choi \\ Information and Communications University, \\ P.O.Box 77, Yusong, Daejeon 305-348 Korea \\ \{twum, yhkwon, jkchoi\}@icu.ac.kr
}

\begin{abstract}
In this paper, we propose an optical burst switching architecture in time division multiplexed wavelength-routing networks, in which an edge OBS node requests time slots necessary to optical bursts to the time division multiplexed wavelength-routing network. Our scheme is attempt to improve the burst contention resolution and optical channel utilization.
\end{abstract}

\section{Introduction}

Researches on the optical internet set a goal of simplified and efficient transmission of IP traffic directly through the WDM layer. The conventional wavelength-routed optical network causes limited scalability and low channel utilization by assigning entire wavelength to a given session. This inefficiency can be reduced by adapting time-slot concepts to the wavelength-routed optical network, in which each individual wavelength is sliced in the time-domain into fixed-length time-slots. Multiple sessions are multiplexed on each wavelength by assigning a sub-set of the time slots to each session.

On the other hand, Optical Burst Switching (OBS) has been proposed as an efficient optical switching method to improve wavelength utilization, in which assembled packets called optical bursts follow a corresponding control packet after an offset time which is latency time required processing the control packets in intermediate OBS routers. Basically, this OBS architecture involves a critical collision problem, which occurs when burst packets contend for the same outgoing interface.

In this paper, we propose a new optical burst switching architecture over time division multiplexed wavelength-routing (TDM-WR) networks, in which an edge node requests time slots necessary to the burst, instead of a whole wavelength. Our scheme can improve the optical channel utilization by sharing the channel as well as it can provide a scalable optical network architecture with a guaranteed QoS. Following this introduction, the proposed network architecture and the control structure are described in section 2 , and the results obtained from simulations will be discussed in section 3. Finally, we draw our conclusions in section 4.

* This work was supported in part by the Korea Science and Engineering Foundation (KOSEF) through the Ministry of Science and Technology (MOST) and Institute of Information Technology Assessment (IITA) through the Ministry of Information and Communication (MIC), Korea. 


\section{Network Architecture}

On the basis of the OBS architecture over TDM-WR networks, which consists of TDM-WR intermediate nodes and OBS edge nodes [Fig. 1], we will investigate the network architecture and control structure in this section.

In the TDM-WR network described in [1], [2] and [3], every network node, which is made up of all-optical switching components, operates with a synchronized timing basis without $\mathrm{O} / \mathrm{E} / \mathrm{O}$ conversion. The wavelengths on their optical links are split into time-slotted wavelengths of a fixed-size interval divided by a time. One or more timeslotted wavelengths within a slotted wavelengths frame can be assigned for a request from an OBS edge node, and a time-slotted wavelength routed path to send the timeslot should be established between the edge OBS nodes by using the control node. On the time-slotted wavelength routed path, each time-slotted optical cross connect (OXC) has a role in switching each slotted wavelength to a destination node. To do this, every time-slotted OXC needs to maintain switching tables containing information entries to forward slotted wavelengths. Fig. 1 shows a time-slot reservation to dynamically control and assign time-slotted wavelength on demand of OBS control packet.

When a data packet arrives at an edge OBS node of the TDM-WR network, the edge node looks up the packet's destination node and pushes it to the corresponding queue to the destination. While the packets are aggregating in the queue, if the queue size reaches given threshold or timeout signal for delay-sensitive data, it has to send a control packet to the control node to request to assign time-slot for the burst. As described in [4], the control node estimates the traffic arrival time from the packet accumulated when the control packet is sent and then establishes the fixed burst size by the time the acknowledgement arrives back at the sending edge router.

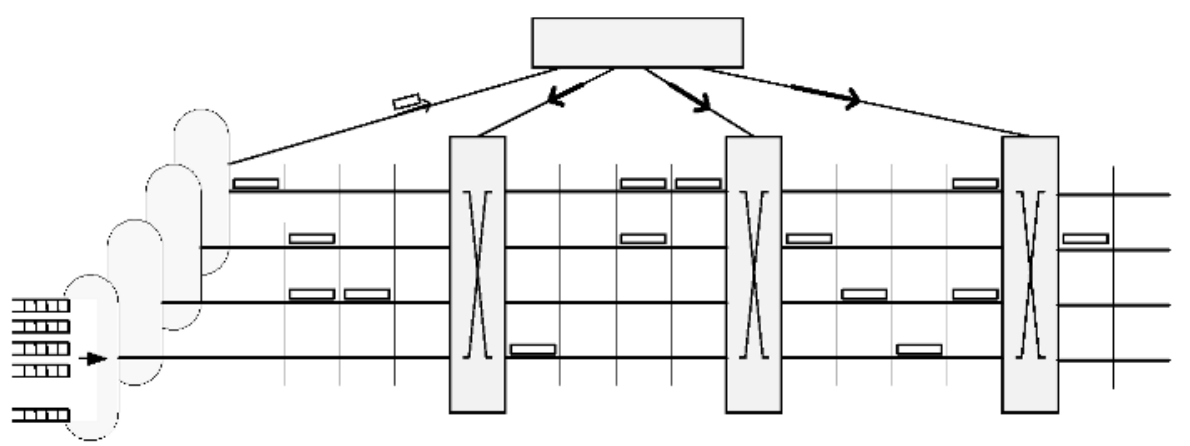

Fig. 1. Control packet delivery to request time-slot

When the control node receives the control packet, it decides whether it can accept this request or not. If, in the requested time-slot, all slotted wavelengths have been used for another request, then the control packet will be rejected. If there are available slots for that request, it will reserve the time slot and reply the edge node with an acknowledgement packet. When the edge OBS node receives the acknowledgement 
packet, it can send the optical burst to the outgoing time-slotted wavelength at the assigned time-slot.

The control plane of the TDM-WR OBS networks encompasses signaling, routing, scheduling, admission control and so on for each optical layer and also requires traffic engineering algorithms to efficiently utilize network resources and to maximize the number of time-slot assigned. To achieve performance objectives, plan of network capacity, selection of the explicit routes, wavelength assignment and timeslot arrangment should be considered.

\section{Simulation Results}

To analyze the proposed OBS scheme, we have developed an OBS simulator by extending NS2 simulator. The network topology and parameters for the simulation are given in Fig. 2. We assume that the average arrival rate is the same for all ingress nodes. Packets arrive at each ingress node according to a Poisson process with $1 \mathrm{Gbps}$ input bit rate. Packets are aggregated into a burst of $1.25 \mathrm{Mbyte}$ size at the ingress node and it is sent to the egress OBS node. The simulation results are obtained for the OBS and our scheme. The performance metrics are link utilization, edge queuing delay as a function of offered input traffic load.

Fig. 3 shows link utilization of the OBS and our OBS scheme using time-slotted wavelength assignment as a function of the offered traffic load per ingress node. Comparing the link utilization of the OBS with the proposed OBS shows that our scheme improves the utilization markedly. In the previous OBS, if an optical burst is collided with another burst, it should be dropped. However, in our scheme, by sending the control packet to the centralized control node, it can prove the available timeslots, if there is not any available time-slot, the edge node will try to reserve next slot again until it succeeds, instead of dropping optical burst. Fig. 4 shows the edge queueing delay versus the offered load. As described in the previous section 2, upon reaching the given threshold value, the edge node sends a control packet and an optical burst in the OBS. So, as the offered load increases, the burst aggregation time decreases and edge queueing delay decreases. However, in our scheme, if there is not available time-slot, the

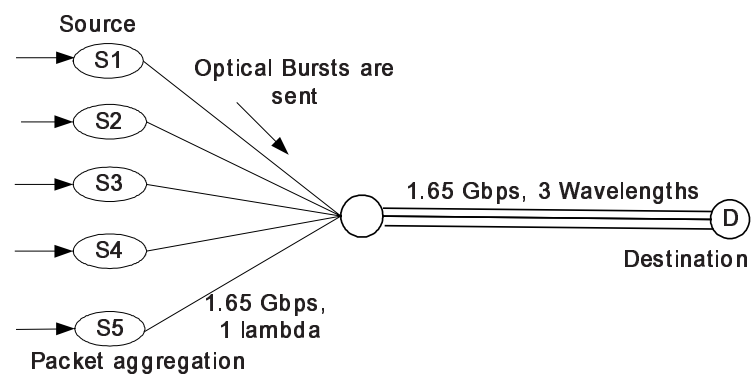

Fig. 2. Simulation topology buffered data should wait until it reserves a time-slot, so it requires more edge delay then the previous OBS. In the previous OBS network, when a burst is blocked, the only way to recover lost packets is TCP retransmission. The TCP makes use of retransmission on timeouts and positive acknowledgments upon receipt of information. However, it could not provide fast recovery due to its host-to-host behavior and time- 
out mechanism. On the other hand, UDP is connectionless, which means that it can not provide error control and flow control.

Therefore, if we consider the TCP/UDP layer's retransmission of the lost packets in the previous OBS, the buffering at the OBS layer in our scheme may support better performance for upper layers. In this paper, we do not analyze the performance of the TCP layer, it will be remained for further studies.

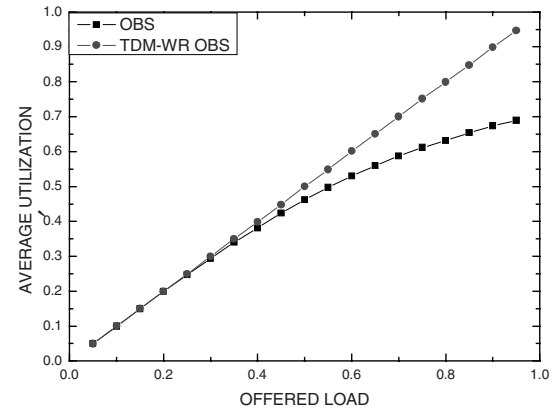

Fig. 3. Offered load vs. Link utilization

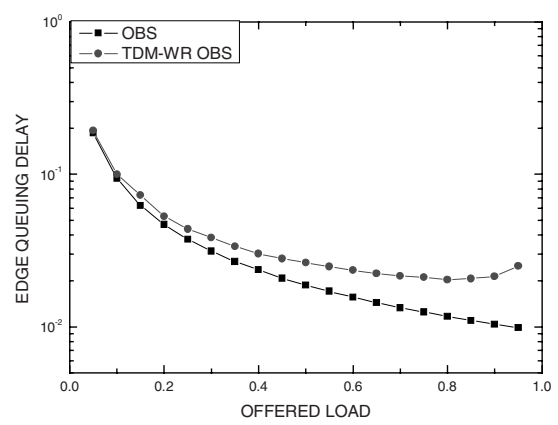

Fig. 4. Offered load vs. Edge queueing delay

\section{Conclusion}

This paper has introduced an optical burst switching over time division multiplexed wavelength-routing network architecture. Our scheme can improve the optical channel utilization by probing the reservation of time-slots as well as it can provide a scalable optical network architecture. Simulation results show that the link utilization of the OBS network is improved markedly at the expense of signaling and edge queueing delay.

\section{References}

1. I.P. Kaminow, et al.: A Wideband All-Optical WDM Network, IEEE Journal on Selected Areas in Communications. Vol. 14 (1996) 780-799

2. Jennifer Yates, David Everitt, and Jonathan Lacey: Blocking in Shared-Wavelength TDM Networks, Australian Telecom. Networks and Applications Conference (1995) 705-710

3. Nen-Fu Huang, Guan-Hsiung Liaw, and Chuan-Pwu Wang: A Novel All-Optical Transport Network with Time-Shared Wavelength Channels, IEEE Journal on Selected Areas in Communications, Vol. 18 (2000) 1863-1875

4. Michael Duser, Polina Bayvel: Anaysis of a Dynamically Wavelength-Routed Optical Burst Switched Network Architecture, Journal of lightwave technologies, Vol. 20, no 4 (2002) 573-585 